\title{
Alice Leflaëc \\ Figures bibliques et idéal familial de la consécration à Dieu
}

\author{
Le protreptique de l'Ad Cytherium (Carm. 24) de Paulin de Nole
}

Aux alentours de 400, ${ }^{1}$ Paulin de Nole, alors retiré depuis quelques années dans son monastère de Nole auprès du tombeau de saint Félix, compose l'Ad Cytherium - le Carmen 24 dans l'édition de von Hartel. ${ }^{2}$ Cette longue lettre en vers iambiques est adressée à un ami lettré et chrétien. Elle relate, dans une première partie, le voyage mouvementé qu'a fait le courrier Martinianus pour se rendre à Nole apporter à Paulin une lettre de Cythérius. La seconde partie du poème, qui nous occupera ici, traite du choix qu'a fait Cythérius de consacrer son fils à la prêtrise. ${ }^{3}$ Le poète, prenant cette décision très au sérieux, adresse à Cythérius et à son fils une série d'exhortations pour que le jeune garçon, alors auprès de Sulpice Sévère, remplisse au mieux sa future charge ecclésiastique. Paulin offre ainsi un "protreptique à la consécration »" et à l'aide de toute une série de figures vétérotestamentaires, il semble dessiner le portrait idéal de l'homme consacré à Dieu au début du $\mathrm{V}^{\mathrm{e}}$ siècle. Si cette figure apparaît en lien étroit avec la vie sacerdotale, nous pouvons remarquer que les exemples bibliques choisis font intervenir des familles entières et donnent ainsi une dimension familiale au thème de la consécration. Paulin semble parler de deux types de consécration en même temps : celle du fils, qui prend la forme d'une vie sacerdotale, et celle, plus générale, qui concerne tout chrétien y compris les parents et qui est en relation avec la pratique de l'ascèse. Par «consécration", nous entendons le fait de se donner à Dieu, ce qui est en lien étroit avec la sanctification, le fait de

1 Fabre (1948) 117-122.

2 Nous utilisons principalement le texte et la classification de la nouvelle édition du Corpus Christianorum : Dolveck (2015). Toutefois, nous nous réfèrerons aussi, entre parenthèses, à la classification de l'édition du CSEL : Hartel (1894). Pour les traductions, voir Ruggiero (1996) et Walsh (1975). Toutes les traductions de Paulin de Nole présentées dans cet article sont personnelles.

3 Sur la structure et l'unité de ce poème, voir Walsh (1976). L'auteur montre le rôle que jouent les exemples bibliques dans l'unité entre les deux parties du poème.

4 Nous ne partageons pas l'avis de G. Guttilla qui considère le Carmen 24 comme une lettre de félicitations adressée à Cythérius qui, avec l'accord de sa femme, a consacré son fils à la vie monastique, et non comme un protreptique adressé à Cythérius ou à son fils (voir Guttilla (1995) 29-30). J. Rougé définit aussi ce poème comme une lettre de félicitation, mais il ne traite pas du tout la seconde partie du poème (voir Rougé (1987) 93). Si le ton de Paulin est, certes, plutôt laudatif et que le poète ne semble pas remettre en cause le salut de Cythérius, il nous semble que les exhortations finales du poème, le souci de Paulin que le fils de Cythérius soit formé au mieux et le thème de la consécration à Dieu invitent à lire la seconde partie de cette composition comme un protreptique adressé à l'ensemble de la famille. Il s'agit, cependant, non pas d'un protreptique à la conversion, mais d'un protreptique «à la consécration». 
rechercher au cours de sa vie la sainteté. ${ }^{5}$ Ce sont les formes de la consécration dans ce poème et les rapports qu'elles entretiennent que nous étudierons, en particulier la manière dont ce protreptique versifié à la consécration est mis en œuvre et le rôle qu'y joue la Bible.

\section{Piété paternelle et consécration de l'enfant}

La seconde partie de l'Ad Cytherium prend, selon l'expression de Serafino Prete, la forme d'une "poetica institutio cleri ${ }^{6}$ », i.e. de l'instruction d'un futur clerc sous une forme poétique. Mais si l'accent est mis sur le comportement que doit adopter le fils de Cythérius, le rôle du père n'est pas pour autant laissé de côté. Au contraire, Paulin de Nole insiste, dans ce poème, sur le rapport entre la piété paternelle et la démarche du fils.

\subsection{Consacrer son enfant à Dieu, un acte de piété et d’obéissance}

L'union de Cythérius et sa femme et d'Anne, la mère du prophète Samuel, dans une même communauté (consortium), nous indique, dans les vers 525-526, que ce sont les parents qui ont décidé de consacrer leur fils à une charge ecclésiastique :

Vobis et Annae sternitur consortium Infantis exemplo sacri :

Samuel et iste creuit in templo Dei, Nunc agnus et pastor dehinc.

\begin{abstract}
Cette communauté s'étend à vous et à Anne par l'exemple de la consécration de votre enfant. Votre Samuel aussi a grandi dans le temple de Dieu, maintenant agneau, il sera bientôt berger.
\end{abstract}

Vers $525-528$

Si ce rapprochement avec Anne est justifié par la ressemblance des situations entre les deux familles, à tel point que Paulin peut parler du fils de Cythérius en employant l'expression Samuel et iste (v. 527), il permet de souligner la foi de Cythérius et de sa femme puisqu'ils sont associés à l'acte de piété d'Anne qui, pour manifester sa reconnaissance à Dieu qui lui avait accordé un enfant malgré sa stérilité, avait décidé de lui consacrer son enfant ( $c f .1$ Sam 1.27-28).

L'identification de Cythérius à Abraham, un peu auparavant dans le poème, fait aussi de la consécration du fils de Cythérius à la vie ecclésiastique un acte d'obéissance de la part de Cythérius :

5 Paulin lui-même n'emploie pas le terme dans ce poème, mais il utilise le verbe consecrare dans l'Epist. 23.10 adressée en 400 à Sulpice Sévère pour évoquer une consécration à Dieu par l'ascèse. Sur les rapports étroits entre le Cyth. et l'Epist. 23, voir Fabre (1948) 119-122.

6 Prete (1964) 82 n. 95. 
Namque ut fideli te patri componeret

500

A te poposcit filium,

In semine Isaac semen ascribens tuum, Ipsumque ut Isaac expetens

Quem tu Abramiae caritatis aemulus

Viuam dedisti uictimam,

505

Deoque tradens iam peremisti tibi

$v t$ saluum haberes firmius.
En effet, pour t'associer au père de la foi, il a réclamé de toi un fils, inscrivant ta descendance dans sa descendance, Isaac, et la désirant elle-même comme Isaac. Et toi, rivalisant d'amour avec Abraham, tu l'as offert comme victime vivante (505) et en le livrant à Dieu, tu l'as fait mourir à toi maintenant pour le garder plus sûrement sauf.

Vers $499-506$

Comme le souligne Guiseppe Guttilla, ce rapprochement avec Abraham est une habitude artistique qui a une finalité laudative implicite. ${ }^{7}$ Il est, en effet, fort positif d'être, selon l'expression du vers 503, l'Abramiae caritatis aemulus. Or, en Gn 22.1-19, c'est par obéissance à Dieu qu'Abraham accepte d'offrir Isaac en sacrifice. En dressant un parallèle avec ce patriarche, Paulin insiste donc sur l'obéissance à Dieu dont a fait preuve Cythérius. Si les parents ont décidé de consacrer leur enfant à Dieu, l'initiative du geste est d'abord divine : «a te poposcit filium» peut-on lire au vers 500.

Le rapport entre l'obéissance et la consécration à Dieu est ici souligné par une réminiscence néotestamentaire : l'allusion à Abraham se double, au vers 504, d'une réminiscence de Rom 12.1 avec l'expression uiua uictima. ${ }^{8}$ Or, il s'agit d'un passage où l'apôtre Paul exhorte clairement ses lecteurs à la consécration. Cythérius fait preuve d'obéissance et de confiance en Dieu lorsqu'il consacre son fils. Il n'hésite pas à offrir son fils en sacrifice par amour à la fois pour Dieu, comme la comparaison avec Abraham le suggère, et pour son fils, puisque, confiant en Dieu, il est convaincu que c'est par ce sacrifice qu'il pourra garder son fils saluus (v. 506). ${ }^{9}$

\subsection{Un fils qui prolonge et accomplit la piété de ses parents}

Cette consécration du fils de Cythérius à la vie sacerdotale n'apparaît, toutefois, pas seulement comme une manifestation de la piété et de l’obéissance de Cythérius. Paulin semble aller plus loin et faire de la consécration du fils le prolongement et l'accom-

7 Guttilla (1995) 18.

8 Paulin superpose aussi cet épisode vétérotestamentaire et cette réminiscence de l'épître aux Romains dans le Nat. 9.616 (Carm. 27), dans un passage où il aspire à être pleinement consacré à Dieu. 9 Nous retrouvons cette idée dans l'Epist. 29.9 adressée en 400 à Sulpice Sévère. Paulin, alors qu'il retrace les grandes lignes de la vie de Mélanie l'Ancienne, évoque le choix qu'elle a fait de se séparer de son fils, convaincue que c'est parce qu'il serait loin d'elle et confié à Dieu qu'elle pourrait le retenir plus solidement.

Malgré la différence de situations - le fils de Cythérius est consacré à la vie sacerdotale, contrairement à celui de Mélanie -, Paulin prend les mêmes exemples parentaux, Anne et Abraham, pour évoquer le geste de Cythérius et celui de Mélanie l’Ancienne. 
plissement de la piété de ses parents. Là encore, ce sont des réminiscences bibliques et l'identification avec des figures vétérotestamentaires qui soulignent cette idée.

Ainsi, le fils de Cythérius se présente comme le signe certain de l'espérance en la vie éternelle de son père. ${ }^{10}$ Après avoir brièvement évoqué l'au-delà (v. 491-494), notre poète s'écrie :

Insigne tantae iam spei certum tibi Magno coruscat pignore!

Plantata Domino in atriis Hierusales Tui propago germinis.
Un signe assuré d'une si haute espérance brille déjà pour toi dans ton précieux enfant ! C'est sur les parvis de Jérusalem qu'a été planté, pour le Seigneur, le rejeton de ta semence.

Vers $495-498$

Or, dans les vers suivants (v. 499-506), Paulin associe le fils de Cythérius à Isaac pour justifier ce propos. ${ }^{11}$ En Genèse, Dieu promet à Abraham qu'il deviendra le père d'une foule de nations (Gn 17.4) et la naissance d'Isaac marque la réalisation de cette promesse. De même, le fils de Cythérius apparaît comme le signe du salut et de la bénédiction de son père.

Ce sont les réminiscences psalmiques des vers 518 à $520^{12}$ qui explicitent le plus cette idée :

Audistis et uos quod beatis dicitur

Vous aussi, vous avez entendu ce qui est dit "Potens erit semen tuum», aux hommes bénis : "Ta descendance sera Habes et illud: "Ventris a fructu tui puissante». Tu connais aussi ceci : (520) «Je In sedibus ponam meis»; placerai dans mes demeures ce qui vient du Qui sermo Dauid, quamlibet Christum sonet, fruit de ton ventre». Or, ces paroles de David, In Christo et illis concinit tout en annonçant le Christ, évoquent aussi Qui christiani corporis collegio In sede ponentur Dei. ceux qui vivent dans le Christ et qui, formant la communauté du corps des chrétiens, seront placés dans la demeure de Dieu.

Vers $517-524$

Si la tradition ${ }^{13}$ lit généralement dans le Psaume 131.11 une annonce du Christ, Paulin, tout en se faisant l'écho de cette lecture au vers 521, offre un élargissement de la compréhension de ce verset. Il concerne aussi les chrétiens qui, selon l'interprétation de notre poète, sont appelés à exercer des fonctions d'autorité à la suite du Christ. Ce passage nous invite alors à voir en Cythérius un homme béni, l'un des beati du vers 517, et à considérer la charge ecclésiastique de son fils comme un accomplissement de cette prophétie.

Cette idée d'un accomplissement de la piété des parents par la vie sacerdotale des enfants est à nouveau exprimée, en quelque sorte, par Paulin à la fin de l'Épi-

10 Cf. Cels. 611-612 (Carm. 31) où Paulin voit dans son défunt fils un espoir de salut, symbolisé par la thématique de la lumière.

11 L'emploi de l'adverbe nam au vers 499 souligne la valeur justificative de l'exemple d'Isaac.

12 Le vers 518 est une réminiscence du Ps 111.2 et les vers 519-520 du Ps 131.11.

13 Cf. notamment Tert., Adv. Marc. III.20 ; Ambr., Epist. 74.22 et in psalm. 1.8. 
thalame (le Carmen 25), un poème composé entre 400 et 404 pour le mariage du futur évêque Julien d'Éclane et de Titia. Le poète exhorte, en effet, les jeunes mariés à pratiquer la chasteté dans le mariage ou, s'ils décident d'unir leurs corps, à donner le jour à un sacerdotale genus (Epith. 237). ${ }^{14}$

Paulin de Nole semble donc lier assez étroitement sa conception de la consécration à la vie sacerdotale et en développer une vision familiale. La continuité généalogique se manifeste, en effet, aussi sur le plan de la foi, les enfants prolongeant la piété de leurs parents. Cela participe, bien sûr, à l'éloge des parents et de leur foi, puisque, comme le fait remarquer Paulin, à propos du fils de Cythérius, seules les racines d'un bon arbre sont fécondes. ${ }^{15}$

\section{Institutio cleri et nouvelle figure de sainteté}

Si la charge ecclésiastique d'un fils constitue l'accomplissement de la piété des parents, l'importance des exhortations adressées à Cythérius et à son fils pour que ce dernier devienne un bon homme d'Église n'en est que plus évidente. Or, pour conforter ses exhortations, Paulin convoque divers exempla vétérotestamentaires qui font émerger, en ce début de $\mathrm{V}^{\mathrm{e}}$ siècle, une nouvelle figure de sainteté.

\subsection{Des exempla vétérotestamentaires au service d'un idéal ascétique de prêtrise}

Notre poète recourt, en effet, à toute une série de figures vétérotestamentaires ${ }^{16}$ pour indiquer au fils de Cythérius le comportement à adopter pour remplir au mieux sa charge. Comme l'a fait remarquer Guttilla, Paulin s'est efforcé de «vivifier d'un point de vue formel l'aridité doctrinale du propos». ${ }^{17}$ Il nous offre, en effet, une composition très soignée où les exempla bibliques s'insèrent dans un portrait du fils de Cythérius et correspondent à diverses étapes de sa vie. Ainsi, Samuel est évoqué pour l'enfance avec la mention de l'éphod tissé par sa mère, Samson pour la jeunesse et les différents apprentissages qu'on y fait et Joseph apparaît à la fin, ses responsabilités administratives en Égypte étant mises en parallèle avec les fonctions sacerdotales qu'aura le fils de Cythérius à l'issue de sa formation. Ce choix de composition révèle l'approche pastorale de Paulin : cherchant à être au plus près des réalités de ses destinataires pour mieux les instruire, il insère, pour ainsi dire, la Bible dans la

14 Epith. 236-237 (Carm. 25) «... quod si corpore congruerint / Casta sacerdotale genus uentura propago.»

15 V. 889-890 «Benedicta prolis sanctae radix ut bonae / Rami feraces arboris».

16 Voir Gnilka (1972) 223-244 sur l'usage par les Pères de l'Église de listes d'exemples bibliques de jeunes gens sages et vertueux.

17 Guttilla (1995) 21. 
vie du fils de Cythérius. S’adressant à des lettrés, ${ }^{18}$ il offre également une composition poétique fort travaillée, ${ }^{19}$ à même de susciter leur intérêt. Il est bien plus plaisant, et dès lors bien plus marquant, de lire un portrait où sont mis en regard âges de la vie humaine et scènes bibliques plutôt qu'une longue liste de préceptes. Ces efforts formels adoucissent aussi le caractère strict des exhortations, car c'est un idéal ascétique de prêtrise qui se dessine dans ce protreptique.

La consécration du fils de Cythérius par ses parents à la vie sacerdotale l'apparente directement aux naziréens de la Bible, ces personnes consacrées à Dieu par un vœu dans le judaïsme. Il n'est donc pas étonnant que Paulin recoure aux exemples de célèbres naziréens de l'Ancien Testament, ici Samuel et Samson, pour mettre en évidence les vertus que le fils de Cythérius doit pratiquer.

Samson, qui n’a pas toujours mené une vie consacrée à Dieu, est loin d'apparaître comme une figure idéale dans la Bible. Pourtant, Paulin lui accorde une large place dans son protreptique (v. 541-588, cf. annexe 1). ${ }^{20}$ Il évoque, en effet, trois épisodes relatés dans le livre des Juges : le lion tué par Samson et dans la carcasse duquel il a trouvé du miel (Jg 14.5-10), le rapport de Samson avec les femmes et avec Dalila en particulier (Jg 14-16) et la mort de Samson et la destruction du temple des Philistins (Jg 16.21-30). Bien conscient de l'ambiguïté de cet exemple, Paulin, qui n’hésite pas à intervenir directement notamment au moyen des verbes nolo (v. 557 et 585) et uolo (v. 581), souligne clairement les enseignements que le fils de Cythérius doit tirer de cet exemple. Ainsi, ce dernier est invité à imiter dans sa vie de prière la puissance dont a fait preuve Samson pour tuer le lion. Mais il doit absolument se garder de la lex carnea (v. 549) qui peut mener à de grands désastres, comme le cas de Samson, qui s'est laissé séduire par des femmes étrangères, l'illustre bien. Ainsi, la succession des substantifs captiuitas, infirmitas et caecitas aux vers 559-560 avec l'enjambement, l'isosyllabisme et les homéotéleutes souligne la chute inéluctable de cet homme. Avec cet exemple, Paulin met en évidence l'opposition topique entre les séductions terrestres et les joies célestes. L'évocation de la mort du naziréen souligne, à nouveau, l'importance de s'opposer au monde. De même que Samson est mort et que sa mort a eu des conséquences positives pour le peuple d'Israël - la destruction du temple des Philistins -, le fils de Cythérius est exhorté à mourir au monde et aux séductions de la chair. ${ }^{21}$ Là encore, le travail poétique met en valeur la

18 Le choix de la forme poétique et non de la prose invite à penser que cette lettre était destinée à une diffusion plus large que Cythérius et sa famille.

19 Le milieu aristocratique possède à cette époque un goût pour le style ciselé, pour le maniérisme. Voir Roberts (1989) 118-121.

20 Samson est à nouveau évoqué dans les vers 605-638. À partir d'une référence discrète au meurtre par Samson de mille Philistins au moyen d'une mâchoire d'âne (Jg 15.15-16) et de la réputation de l'âne comme animal paresseux ( $c f$. Apul., Met.VI.31 et VII.21 et Ambr., Hex.VI.3.11), le poète exhorte le fils de Cythérius à faire preuve de courage, à tuer toute paresse en lui et à vaincre ainsi la chair et ses séductions.

21 Paulin de Nole offre également une méditation sur l'épisode de Samson dans l'Epist. 23. Si son interprétation ne diffère guère de celle du Cyth., Paulin fait, cependant, de la mort de Samson une 
recommandation : dans les vers 582-583 («Sic morte mortem, ut permanens / In carne carnem uincat et uiuat Deo»), les polyptotes morte / mortem et carne / carnem, eux-mêmes accentués par la juxtaposition des termes, mettent en évidence le caractère oxymorique et difficile à réaliser de l'exhortation. Or, ce thème d'une séparation radicale avec le monde et ses attraits est ascétique et se trouve à maintes reprises dans la poésie de Paulin. ${ }^{22}$

L'idée d'une lutte contre la chair est aussi illustrée par l'exemple de Joseph, ${ }^{23}$ où le poète insiste sur la chasteté et la maîtrise de soi dont a fait preuve le patriarche au cours de sa vie :

Sit et hic probatus corporis custos sui, $V t$ claustra teneat carceris ;

Si uitia carnis strinxerit Christi metu, Quasi uinculatis praeerit, Aliisque mundi carceri inclusis adhuc Et alligatis saeculo, Vt liber ipse iamque commissus sibi, Magister et custos erit.

\begin{abstract}
Que notre enfant aussi soit éprouvé comme gardien de son corps (750) afin qu'il garde les portes de la prison. $\mathrm{Si}$, par la crainte du Christ, il arrache les vices de la chair, il sera comme à la tête de ceux qui sont enchaînés; et pour les autres, encore enfermés dans la prison du monde et attachés au siècle, (755) parce qu'il sera lui-même libre et désormais confié à luimême, il sera un maître et un gardien.
\end{abstract}

Vers $749-756$

Le fils de Cythérius est invité à être éprouvé, de la même manière, comme gardien de son corps afin de prouver sa capacité à se maîtriser et ainsi à conduire les fidèles. ${ }^{24}$

C'est dans le traitement de l'exemple de Joseph que se trouve résumé le principe directeur de cette institutio cleri : «Caeli uitam in terris age» (v. 804). Le futur prêtre doit vivre les yeux tournés vers le ciel. Nous conservons ici la leçon uitam de von Hartel, contrairement à Dolveck qui édite uiam. ${ }^{25}$ Cette leçon invite à voir dans ce vers une réminiscence d'une expression de la Vita Pauli de Jérôme, «cum [...]beatus Paulus uitam coelestem ageret in terris» (7.22.10). Paulin ferait donc allusion à un texte qui fait l'éloge de la vie ascétique et même érémitique.

Dans les vers 515-516, la réminiscence du Psaume 70, 6 associe le fils de Cythérius à une autre figure vétérotestamentaire, le roi David.

préfiguration de celle du Christ (Epist. 23.13). L'exégèse allégorique pratiquée par Paulin est rare. $C f$. Ambr., Epist. 62.8-34 (éd. maur. 19) où Ambroise se sert de l'interprétation littérale de l'histoire de Samson pour montrer qu'il faut se garder des mariages avec les étrangers.

22 Cf. notamment, Nat. 13.428-459 (Carm. 21) et Cels. 447-578 (Carm. 31).

23 Le poète n'évoque pas seulement les naziréens de l'Ancien Testament. Le fils de Cythérius est aussi associé à Isaac, à Joseph et à David.

24 Cf. Ambr., Epist. e. c. 14.7 (éd. maur. 63). Augustin souligne aussi l'importance pour l'orateur de conformer sa vie à ses paroles s'il veut se faire écouter avec docilité (Doct. chr. 4.27.59-60).

25 W. von Hartel indique que uiam est une leçon du ms B (Codex Bruxellensis 10615-10729), F. Dolveck ne donne rien dans son apparat à ce sujet, mais il indique que la traditio textus pour ce passage sont les mss $S, J, B$. Il explique également dans son introduction (Dolveck (2015) 124) que $B$ est le résultat d'une contamination et qu'il est assimilé au groupe que forment avec lui les mss $S, J, O$ et $V$. 
Et nunc in aula paruulus ludit Dei Et ore lactanti canit : "De uentre matris et die prima mihi Tu, Christe, protector meus !»
Et à présent, le petit enfant joue dans la cour de Dieu et, de sa bouche nourrie par le lait, chante : «Dès le ventre de ma mère et dès mon premier jour, toi, ô Christ, tu es mon protecteur !».

Vers $513-516$

La louange de Dieu au moyen des psaumes apparaît donc comme une forme de consécration à Dieu. Avec cette réminiscence, Paulin décrit le fils de Cythérius comme chantant les Psaumes depuis sa plus tendre enfance. Or, comme le précise Guttilla, ${ }^{26}$ ce chant des psaumes dès l'enfance est un motif topique que l'on trouve chez Jérôme ${ }^{27}$ et en l'utilisant, notre poète s'inscrit dans la tradition littéraire du protreptique à la sanctification.

L'Ad Cytherium est composé à une époque où se développe, notamment avec la Vita Martini de Sulpice Sévère, le modèle du moine-évêque. ${ }^{28} \mathrm{Il}$ s'agit à la fois d'un texte bien connu de Paulin et d'un modèle qu'il approuve - lui-même, moine, deviendra évêque vers 410 . Or, il semblerait qu'avec notre poème, Paulin, en recourant à diverses figures vétérotestamentaires, participe à l'élaboration et à la diffusion de ce modèle en lui donnant des assises bibliques.

En lisant cette liste d'exemples bibliques, le lecteur peut, néanmoins, se poser une question : pourquoi, alors qu'il insiste, dans les vers 829-830 notamment, sur l'importance de connaître toute la Bible, ${ }^{29}$ Paulin n'évoque-t-il que des figures vétérotestamentaires, laissant de côté, par exemple, le personnage de Jean-Baptiste dont la manière de vivre correspond pourtant à son propos?

\subsection{Une nouvelle étape dans la manière de vivre la consécration}

S'il n'est pas simple de répondre à cette question, le survol de quelques étapes de ce protreptique met en avant l'importance d'une opposition forte entre la vie selon la chair et celle selon l'esprit. Pour cela, Paulin opère une spiritualisation des actions des figures de l'Ancien Testament pour les chrétiens de son époque. Ainsi, le fils de

26 Guttilla (1995) 19 n. 61.

27 Jer., Epist. 107.4 adressée à Laeta pour l'instruction de sa fille Paula : «Adhuc tenera lingua psalmis dulcibus imbuatur».

28 Grand ami de Sulpice Sévère, Paulin de Nole a fortement contribué à la diffusion de la Vita Martini en Italie et en Illyrie (cf. Sulp. Sev. Dial. 3.17). Sur les rapports entre les deux hommes et leur correspondance, voir Desmulliez et al. (2016).

29 Voir Cyth. 827-830 :

In carne uiuens, uita carnis exsulet

In lege mentis ambulans,

Totamque regni crediti terram sibi

Peragret in Libris sacris. 
Cythérius est exhorté à imiter la puissance de Samson par ses prières et non en partant à la chasse au lion...

Précisément, Paulin explique ce principe de lecture de l'Ancien Testament dans les vers $639-642$ :

Sed quae ante sanctis in figuram gesta sunt Nobis in actum scripta sunt, $V t$ quod parentes gestitarunt corpore Nos actitemus spiritu.
Mais ce que les saints ont accompli auparavant en figure a été écrit pour nous en acte, de sorte que ce que nos parents ont d'ordinaire accompli dans leur corps, nous ayons l'habitude de le faire, nous, en esprit.

En s'appuyant sur une réminiscence de 1 Cor 10.11, le poète oppose deux époques, celle des patriarches et la sienne, auxquelles sont associées deux manières d'accomplir les mêmes actions : les patriarches les font corpore, les chrétiens spiritu. Ce déplacement est le fruit de l'œuvre de salut du Christ, explique Paulin dans les vers suivants. $^{30}$

Mais le poète ne se contente pas d'énoncer ce principe exégétique. Dans son usage des différentes figures vétérotestamentaires, il le met en application. ${ }^{31}$ Par exemple, à la lecture littérale du récit de la vie de Samson s'ajoute une interprétation allégorique dont Paulin donne la clé de lecture au v. 549 : «Allophyla mulier est mihi lex carnea». La femme étrangère du livre des Juges représente, en fait, une entité bien plus grande et dangereuse, la loi de la chair symbole du péché.

Peut-être Paulin se concentre-t-il sur les figures vétérotestamentaires dans une optique exégétique et pastorale : il se soucie de la bonne compréhension de l'Ancien Testament du fils de Cythérius, parce que ce dernier devra enseigner les fidèles. ${ }^{32}$

Mais nous pensons surtout que la manière dont notre poète traite les différentes figures vétérotestamentaires insiste sur une spiritualisation de la lutte entre le bien et le mal qui vient renforcer le portrait de l'homme consacré dessiné par Paulin. En ce début de $\mathrm{V}^{\mathrm{e}}$ siècle, cet homme n'est plus forcément persécuté, voire destiné au martyre. Le combat pour la consécration à Dieu n'en est pas, pour autant, terminé, mais il prend une nouvelle forme. Ainsi, au vers 753, l'image de la prison du monde se substitue à celle matérielle de Joseph. ${ }^{33}$ Il s'agit, pour le fils de Cythérius, d'une lutte entre la chair et l'esprit, entre les séductions terrestres et les bénédictions célestes. Là encore, Paulin participe à la diffusion du modèle ascétique en lui donnant des fondements bibliques et en montrant que la consécration à Dieu ne

30 Voir les vers 643-668 où l'influence paulinienne est manifeste, en particulier par des réminiscences des deux épîtres aux Corinthiens.

31 Cf. Nat. 9 (Carm. 27), où Paulin accorde également une grande importance à l'exégèse allégorique de plusieurs passages scripturaires, notamment de l'histoire de Ruth (v. 529-541).

32 Dans les vers 669-688, Paulin, en insistant sur le rôle de chacun des Testaments, exhorte le fils de Cythérius à se nourrir des Écritures pour se préserver des dogmes nuisibles.

33 "Aliisque mundi carceri inclusis adhuc». 
revêt pas toujours les mêmes formes d'une époque à l'autre. Après le temps du martyre vient celui de la consécration à Dieu par l'ascèse.

Le recours aux exempla vétérotestamentaires permet donc de définir un idéal chrétien de la consécration pour un futur prêtre au début du $\mathrm{V}^{\mathrm{e}}$ siècle, un idéal marqué par les tendances ascétiques qui se développent à cette époque. ${ }^{34}$ Mais la lecture de l'Ad Cytherium montre que ce n'est pas seulement à la consécration du fils de Cythérius que s’intéresse Paulin. Il nous présente, en effet, une véritable économie familiale de la consécration.

\section{Une économie familiale de la consécration}

\subsection{Une aide réciproque}

En recourant non seulement à des figures particulières de l'Ancien Testament, mais aussi à des familles vétérotestamentaires, le poète met en évidence le rôle des parents dans la consécration de leur enfant. ${ }^{35}$ Cependant Paulin, au moyen de l'exemple de Joseph, développe également l'idée d'un rôle du fils dans la consécration de ses parents.

Vt ille quondam prouidentia Dei

Distractus in seruum puer

Ad arua frugum missus est ut et patrem

Praeiret et fratres suos,

Qui mox, per orbem consecutura fame,

Patris altor et fratrum foret.

Sic iste forsit in sacram panis domum

Vos antecessit filius

$V t$ et parentes pascat et fratres suos

In istius mundi fame,

Vbi terra, tribulos parere nobis largior,

Eget bonorum frugibus.

\begin{abstract}
Comme autrefois, le fameux enfant, par la providence divine, fut vendu comme esclave et envoyé vers les champs de céréales, pour précéder à la fois son père et ses frères, (705) lui qui bientôt, lors de l'arrivée imminente d'une famine sur la terre, serait celui qui allait nourrir son père et ses frères, de même, votre fils vous a peut-être devancés dans la demeure sacrée du Pain pour nourrir ses parents et ses frères (710) durant la famine de ce monde, là où la terre est plus prodigue à nous susciter des ronces et a besoin des moissons des hommes de bien.
\end{abstract}

Vers $701-712$

34 Ambroise, dans l'Epist. e. c. 14.66 (éd. maur. 63) datée de 396, souligne la nécessité d'avoir un évêque qui mêle sévérité monastique et discipline ecclésiastique pour l'Église de Verceil.

35 Paulin de Nole ne compare pas seulement Cythérius à Abraham. Il associe également son épouse à Anne, la mère de Samuel qui tisse un éphod pour son fils. La mère participe ainsi à la consécration de son fils en prenant, matériellement, soin de lui. Les vers 530-532 invitent aussi à une interprétation allégorique du vêtement. Faut-il en conclure que l'éphod que la mère de Cythérius doit coudre pour son fils correspond, en fait, à l'instruction religieuse qu'elle doit lui dispenser ? Les vers 689 à 696 soulignent, en tout cas, le rôle que joue l'épouse de Cythérius auprès de son mari ainsi que dans la croissance spirituelle de ses enfants. "In castitate liberos enutriens / Vitam nouellantes Deo» (v. 695-696). 
Dans ces vers, Paulin effectue un parallèle entre Joseph, qui a précédé sa famille en Égypte afin de la sauver d'une famine, et le fils de Cythérius, qui a devancé ses parents dans la sacra panis domus (v. 707) afin de peut-être les nourrir spirituellement au milieu l'istius mundi fames (v. 710). Selon une étymologie courante, ${ }^{36}$ l'expression sacra panis domus désigne Bethléem, haut-lieu d'ascétisme. Mais l'évocation de la famine du monde montre que Paulin donne aussi un sens métaphorique à l'expression sacra panis domus et qu'elle désigne également la cité de Dieu. Dans le contexte de l'instutio cleri, il faut peut-être aussi y voir une allusion à l'Église et l'Eucharistie que distribuera le fils de Cythérius une fois ordonné prêtre. Avec cette périphrase, le poète est à l'œuvre et souligne la double localisation du fils de Cythérius, sa position terrestre, dans l'Église, reflétant pour ainsi dire une réalité céleste. Par sa vie sacerdotale, le fils de Cythérius précède donc ses parents dans la demeure de Dieu. Il est, comme l'écrit Paulin au vers 880, leur salutis praeuius et peut ainsi agir pour leur bien en les nourrissant spirituellement parce que lui-même est nourri par Dieu. ${ }^{37}$

Paulin joue sans doute ici avec le thème du puer senex, ${ }^{38}$ de l'enfant jeune par les années mais âgé par sa sagesse. En effet, l'enfant est pour ses parents un magister paruulus (v. 894). Plus généralement, ce rôle du fils pour les parents relève de l'intercession et rappelle celle que les défunts qui ont atteint un haut degré de sainteté, qu'ils soient morts en martyrs ou non, exercent en faveur des vivants encore en proie aux luttes terrestres. Rappelons que c'est par ses prières que le fils de Cythérius est appelé à imiter la puissance de Samson. Paulin développe cette idée d'intercession dans le De obitu Celsi (le Carmen 31) : il invite en effet deux défunts qui partagent le même nom de Celsus, son propre fils et le fils des amis auxquels il adresse sa consolation, à prier pour le salut de leurs parents encore vivants. Préservés du mal du monde par leur mort précoce, ces enfants se trouvent dans le séjour de Dieu et peuvent prier avec efficacité pour la rémission des péchés de leurs parents. ${ }^{39}$

La différence, dans l'Ad Cytherium, réside dans le fait que c'est encore vivant que le fils de Cythérius pourra agir pour le bien spirituel de ses parents. Avec ce développement sur le rôle du fils de Cythérius et le recours à l'exemple de Joseph, Paulin

36 Voir Ambr., Iac. II.7 ; Epist. 18.9 ; 19.2 et Jer., in Ier. VI.19 et in Mich. II.5.

37 Voir vers 891-893 :

Deo dedistis nutriendum filium,

Et ille sic uobis alit

Vti uicissim nutriat canos puer.

Ce rôle du fils vis-à-vis d'un père déjà chrétien peut surprendre, mais le poète semble suggérer l'idée que le salut et la sanctification sont un trava il quotidien.

38 Voir Curtius (1956) 122-125 et Gnilka (1972), en particulier 135-147 sur le rapport entre cet idéal et le mouvement ascétique.

39 Voir Cels. 591-628 (Carm. 31), en particulier les vers 617-618 :

Innocuisque pares meritis peccata parentum,

Infantes, castis uincite suffragiis ! 
montre donc que l'efficacité «spirituelle» et plus précisément celle de l'intercession concerne, non pas les seuls martyrs ou les défunts particulièrement saints, mais plus généralement les hommes véritablement consacrés à Dieu, et donc en premier lieu ceux qui remplissent une charge ecclésiastique.

\subsection{Un renversement des rôles}

Dans notre extrait, c'est même un véritable renversement des rôles qui s'opère, le fils de Cythérius étant, au vers 896, factus parens parentibus. Paulin insiste beaucoup sur cette idée de renversement en insérant, au milieu de l'exemple de Joseph, une longue comparaison avec le comportement des aigles, où l'on peut observer une alimentation des parents par les jeunes aigles. ${ }^{40}$ Un tel développement sur le thème du renversement des rôles et des bienfaits spirituels peut surprendre, et le poète luimême parle d'un pietatis admirabile mysterium (v. 895). Mais il montre que la dimension familiale de la consécration n'est pas à sens unique : si les parents agissent pour la consécration de leur enfant, ce dernier, parce qu'il est devenu un modèle de sainteté, peut à son tour agir pour leur bien et les aider à parvenir, eux aussi, dans la demeure éternelle de Dieu. Cette vision familiale de la consécration est liée à l'idéal de l'ascète en charge de fonctions ecclésiales, mais elle le dépasse aussi puisque la consécration de tous est recherchée. Et de fait, le poème se termine sur des exhortations ascétiques adressées directement à Cythérius et sa femme, telles que se débarrasser du poids des possessions et de la souillure du péché pour être prêts à rencontrer Dieu.

Dans l'Ad Cytherium, une composition fort travaillée adressée à des lettrés, Paulin de Nole recourt à toute une série de figures vétérotestamentaires pour illustrer le comportement que doit adopter un futur prêtre. Il montre ainsi toute la valeur normative qu'il accorde à la Bible. Mais cette dernière est aussi utilisée dans le contexte du christianisme du début $d u V^{e}$ siècle où se diffuse de plus en plus la figure du moine-évêque. Avec l'institutio cleri présente dans la seconde partie de ce poème, Paulin donne des assises bibliques au personnage du prêtre ascète et fait de lui une figure idéale de consécration. Toutefois, avec les différents personnages vétérotestamentaires qu'il évoque, Paulin souligne, et c'est peut-être là l'aspect le plus singulier de notre passage, le caractère familial de la consécration : si les parents agissent pour le bien de leur enfant en le consacrant à Dieu, le fils accomplit pleinement la piété de ses parents et même, grâce au rôle qu'il acquiert, peut œuvrer pour leur salut afin qu'ils atteignent eux aussi le Royaume de Dieu. Or, c'est par la

40 Il s'agit des vers 851-884. Le poète lui-même insiste sur la valeur exemplaire des oiseaux qui parlent spontanément des «regni caelitis mysteria» (v. 873). 
consécration à Dieu qu'il est possible de l'atteindre. Si le fait de se donner à Dieu ne revêt pas forcément la forme d'une charge ecclésiastique, il suppose, néanmoins, un rejet des valeurs terrestres et un engagement radical pour le Christ. En somme, sur le chemin de la sanctification, c'est à l'ascèse qu'invite notre poète.

\section{Annexe : Vers 541-588 L'exemple de Samson}

Et, ut ille Samson ui capillorum potens, Virtute crinitus sacra

Sternat leonem strangulatum fortibus

Orationum bracchiis,

545 Dulcemque fructum nobilis uictoriae

Decerpat ore mortui.

Sed ab hoc triumpho caueat exemplo uiri

Aliena adire foedera ;

Allophyla mulier est mihi lex carnea

550 Blandis dolosa retibus :

Si lege mentis ista sit lex fortior,

In iura peccati trahet,

Malesuada uerbis fraudis arte dulcibus

Animum uirilem effeminat,

555 Excaecat oculos mentis et radit caput, Spolians et exarmans fidem.

Hac parte Samson nolo sit noster puer,

Ne misceatur copulae,

Quam consequatur protinus captiuitas

560 Infirmitas et caecitas,

Licet ille fortis postea receperit

Robur recretis crinibus,

Manuque ductus de mola ad ludibrium

Hostilis exsultantiae,

565 In caecitate corporis mente intuens

Vocarit ultorem Deum ;

Et, restituto mox capillis robore,

Prostrauit hostilem domum,

Cuius columnas fortior saxis manus

570 Vt clausit amplexu graui, Collapsa fulcris tecta subductis humo

Cecidere in ipsum ; sed tamen

Et morte <in> ipsa praepotens heros Dei

Hostes ruinae miscuit

Et gloriosa morte pensauit sibi

575 Vitae subactae dedecus :

Qui seruus hoste gloriante uixerat

Hoste obruto uictor cadit,

Et plura moriens interemit milia,

Quam uiuus interfecerat.

580

Imitetur istam filius noster uolo
Et comme le fameux Samson, dont la force des cheveux le rendait puissant, doté à son tour d'une puissance sacrée dans ses cheveux, qu'il terrasse le lion étranglé par les bras vigoureux de ses prières (545) et qu'il recueille le doux fruit d'une noble victoire dans la bouche d'un mort. Mais à partir de l'exemple de ce triomphe, qu'il se garde de conclure des alliances avec des étrangères. La femme d'un autre peuple est, pour moi, la loi de la chair, (550) qui piège dans ses rets séduisants. Si cette loi est plus forte que celle de l'esprit, elle l'entraînera dans les pouvoirs du péché ; persuadant le mal avec de douces paroles selon l'art de la tromperie, elle effémine l'esprit viril, (555) elle aveugle les yeux de l'esprit et rase la tête en dépouillant et désarmant la foi. Je refuse que notre cher enfant soit un Samson à cet égard, de peur qu'il s'engage dans une union que suivront aussitôt la captivité, (560) l'infirmité et la cécité, bien que cet homme vigoureux ait recouvré, par la suite, sa puissance, lorsque ses cheveux repoussèrent, et que, conduit par une main depuis son moulin pour être la risée de ses ennemis en liesse, (565) voyant par son esprit malgré sa cécité physique, il ait appelé la vengeance de Dieu. Et comme sa force lui fut bientôt rendue par ses cheveux, il renversa la demeure ennemie ; et, quand sa main, plus forte que les rochers, (570) enferma les colonnes dans une étreinte violente, les montants s'arrachèrent du sol, les toits s'écroulèrent et tombèrent sur lui ; et, cependant, le héros de Dieu, puissant même dans sa mort, mêla ses ennemis à sa chute (575) et il compensa par sa mort glorieuse le déshonneur d'une vie d'assujettissement. Lui qui avait vécu comme esclave sous un ennemi qui se glorifiait tomba victorieux en écrasant l'ennemi et, en mourant, il fit périr plus de milliers (580) qu'il en avait tué durant sa vie. Je veux que notre cher fils 
Sic morte mortem, ut permanens In carne carnem uincat et uiuat Deo, Peccata carnis opprimens ; Sed nolo carnis gaudiis ut noxiae 585 Dolis subactus feminae Addicat animum, et praeda fiat hostium, Virtute nudus gratiae. imite cette mort par sa mort, pour qu'en demeurant dans la chair, il puisse vaincre la chair et vivre pour Dieu en écrasant les péchés de la chair. (585) Mais je refuse qu'il voue son esprit aux plaisirs de la chair, comme soumis aux ruses d'une femme nuisible, puis qu'il devienne la proie des ennemis, dépouillé de la puissance de la grâce.

\section{Bibliographie}

Curtius (1956): Ernst Robert Curtius, La Littérature européenne et le Moyen Âge latin (transl. Jean Bréjoux), Paris.

Desmulliez et al. (2016): Janine Desmulliez, Cédric Vanhems and Jean-Marc Vercruysse, Paulin de Nole Correspondance avec Sulpice Sévère, Paris.

Dolveck (2015): Franz Dolveck, Paulini Nolani Carmina, Turnhout.

Fabre (1948): Pierre Fabre, Essai sur la chronologie de l'œuvre de Saint Paulin de Nole, Strasbourg.

Gnilka (1972): Christian Gnilka, Aetas spiritalis. Die Überwindung der natürlichen Altersstufen als Ideal frühchristlichen Lebens, Bonn.

Guttilla (1995): Guiseppe Guttilla, «ll carme 24 di Paolino di Nola e la sua novitas», in: Koinonia, $19,1,5-31$.

Hartel (1894): Wilhelm von Hartel, Sancti Pontii Meropii Paulini Nolani Carmina, Vienne.

Prete (1964): Serafino Prete, Paolino di Nola e l'umanesimo cristiano : saggio sopra il suo epistolario, Bologna.

Roberts (1989): Michael Roberts, The Jeweled Style. Poetry and Poetics in Late Antiquity, Ithaca London.

Rougé (1987): Jean Rougé, «Un drame maritime à la fin du Ve siècle : le voyage de Martinien de Narbonne à Nole (Paulin, poème 24)», in: Jean-Marie Pailler (ed.), Mélanges offerts à monsieur Michel Labrousse, Toulouse, 93-103.

Ruggiero (1996): Andrea Ruggiero, Paolino di Nola. I carmi. Testo latino con introduzione, traduzione italiana, note e indici, Napoli Roma.

Walsh (1975): Patrick Gerard Walsh, The poems of St. Paulinus of Nola, New-York.

Walsh (1976): Patrick Gerard Walsh, «Paulinus Nolanus, Carmen 24», in: Joseph O’Meara and Bernd Naumann (eds.), Latin script and letters A.D. 400-900. Festschrift presented to Ludwig Bieler on the occasion of his 70th birthday, Leiden, 37-43. 